\title{
Übertragung heilkundlicher Tätigkeiten nach § 63 Abs. 3c SGB V am Beispiel Wundmanagement
}

Martina Roes

(C) Springer-Verlag Wien 2012

Mit der Verabschiedung des Gesetzes zur strukturellen Weiterentwicklung der Pflegeversicherung können im Rahmen der Gesetzlichen Krankenversicherung ( $\S 63 \mathrm{ff}$. SGB V) Modellvorhaben realisiert werden. Damit würde für Pflegende die Ausübung heilkundlicher Tätigkeiten eingeführt werden.

In einer Arbeitsgruppe des Gemeinsamen Bundesausschusses (GBA), an der auch Vertreter/innen des Deutschen Pflegerates (DPR) mitwirkten, wurde u. a. das Thema „Versorgung und Management chronischer Wunden“ vorgeschlagen und in die 2011 vom GBA verabschiedete Richtlinie aufgenommen.

Nach erfolgreichem Abschluss der im $\S 63$ ff. SGB V vorgeschriebenen Modellprojekte könnte „Versorgung und Management chronischer Wunden" als Vorbehaltstätigkeit der Profession Pflege definiert werden, für die Ärzte nicht speziell ausgebildet werden. Vor diesem Hintergrund stellen sich folgende Fragen: 1) welche Qualifikationsbedarfe ergeben sich hieraus für die Pflegenden, 2) welche Änderungen hinsichtlich derzeitiger Rahmenbedingungen sind notwendig und 3) wie kann eine zukunftsfähige interdisziplinäre Zusammenarbeit aussehen?

Analysiert und reflektiert werden u. a. derzeitige Fortbildungs-/Zertifizierungsmodule ,Wundmanager/in ' unterschiedlicher Anbieter mit Blick auf deren Kompetenzprofile; Einordnung des als notwendig erachteten Kompetenzlevels in der Bachelor und Master Struktur; sowie Kopplung der Kompetenzlevel an Verantwortungsbereiche (u. a. Delegation/Substitution).

Vorherrschende, auf Separation ausgerichtete, professionstheoretische Ansätze (die auf Konzepten wie ,social closure ' und ,professional authority' basieren) müssen kritisch reflektiert werden. Eine Diskussion darüber, welche innovativen Ansätze benötigt werden, um die Entwicklung eines zukunftsfähigen, interprofessionellen Selbstverständnisses zu unterstützen, ist notwendig.

M. Roes $(\triangle)$

Leiterin der Arbeitsgruppe Implementierungsund Disseminationsforschung, Deutsches Zentrum für

Neurodegenerative Erkrankungen (DZNE), Witten, Deutschland 